\title{
Combining Ability Analysis: Morphological Traits for High Temperature Stress Tolerance in Indian Mustard [Brassica juncea (L.) Czern \& Coss.]
}

\author{
Baldeep Singh*, N.K. Thakral, Ram Avtar and Geeta Boken \\ Department of Genetics and Plant Breeding, CCS Haryana Agricultural University, \\ Hisar- 125004, India \\ *Corresponding author
}

\begin{abstract}
A B S T R A C T
Half diallel analysis of eight parents was carried out to identify the high heterotic crosses and their relationship in terms of general and specific combining ability (gca \& sca) in Brassica juncea L. Czern and Coss. Mean squares due to parent v/s crosses were also significant for all the traits which depicted presence of heterosis for all the traits, except for days to maturity, plant height, siliquae on main shoot, number of seeds/siliqua, siliqua

\section{Keywords}

Brassica juncea, Additive, gca, sca, Yield components.

Article Info

Accepted: 12 April 2017 Available Online: 10 May 2017 length and oil content in timely sown condition and for plant height, number of primary branches per plant, siliqua on main shoot and 1000 seed weight in late sown condition. The heritability in narrow-sense showed the prevalence of additive variance for siliqua length, days to maturity, number of primary branches per plant in late sown condition, while for other traits an appreciable proportion of total variance was non-additive in both the environments. In the present study, an overall appraisal of GCA effects revealed that RH0735 and BPR349-9 in normal environment and RH0116 and RH0555A in late sown environment were good general combiner for majority of the characters. High GCA effects are related to additive gene effects or additive $\mathrm{x}$ additive interaction which represent the fixable genetic component of variation. Hence these parents could be efficiently used for exploiting seed yield. For seed yield the crosses RH8814 x RH0555A, RH0644 x BPR5433 and BPR349-9 x RH0644 in timely sown condition and crosses RH0555A x RH0644, RH0735 x RH0116 and BPR349-9 x RH0644 were identified as promising on the basis of their high per se performance and with high significant SCA effects. These crosses could be extensively used in breeding programme to develop superior segregants and the parents involved may be converted to well adapted cytoplasmic male sterile or restorer lines in further breeding programmes.
\end{abstract}

\section{Introduction}

Indian mustard (Brassica juncea) is a naturally autogamous species, yet in this crop frequent out-crossing occur which varies from 5 to $30 \%$ depending upon the environmental conditions and random variation of pollinating insects. Cytologically Indian mustard is an amphidiploid $(2 \mathrm{n}=36)$, derived from interspecific cross of Brassica campestris $(2 \mathrm{n}=20)$ and Brassica nigra $(2 \mathrm{n}=16)$ followed by natural chromosome doubling. These relationships have been confirmed by the artificial synthesis of amphidiploids species by hybridizing basic diploid species and also by analysis of chloroplast and mitochondrial DNA restriction pattern of basic and amphidiploids 
species. The improved mustard seeds contain $39-44 \%$ oil. For International acceptance, erucic acid content should be $<2 \%$. In India the area of rape and mustard 5.7 Mha, Production 5.74 MT and yield $1007 \mathrm{~kg} / \mathrm{ha}$ in 2014-15, rapeseed-mustard has now become the second largest produced edible oilseed crop in the world after soybean (FAO, 2013).

For developing a hybrid, as a first step information available on genetic analysis of important characters is collected. This information are then used to combine desirable traits in a single hybrid. For this purpose, genetic information on heterosis is useful for developing breeding strategies to meet the demands of increased population. To estimate nature and magnitude of general combining ability (additive gene actions) and specific combining ability (non-additive gene actions), two approaches are very common i.e. top-crosses and diallel crosses for conducting a successful breeding program (Amiri-Oghan et al., 2009).

Estimation of genetic constitution of parents for seed yield and it components can be important for indirect selection for high seed yield in rapeseed (Nassimi et al., 2006; Singh et al., 2010). Although combining ability studies in oilseed Brassica are scanty, most of these studies emphasized the preponderance effect of gca for yield and its components indicating the importance of additive gene action (Wos et al., 1999). On the other hand, Teklewold et al., (2005) reviewed evidences for the presence of significant sca effects for yield and yield components. Ramsay et al., (1994) reported that variation for both gca and sca were responsible for dry matter yield and other quantitative traits in $B$. napus. Significant gca and sca effects were reported for siliquae per main shoot, siliquae per plant, siliqua length, number of seeds per siliqua, 1000-seed weight and seed yield in B. napus (Leon, 1991; Thakur and Sagwal, 1997).

\section{Materials and Methods}

This study was carried out at the research area of the Oilseeds Section, Department of Genetics \& Plant Breeding, CCS HAU, Hisar $\left(29^{\circ} 10 \mathrm{~N}^{\prime}\right.$ lat., $75^{\circ} 46^{\prime} \mathrm{E}$ long., $215 \mathrm{~m}$ alt.) during 2013-2015. Eight diverse mustard genotypes namely RH8814, RH0735, RH0116, BPR349-9 (Tolerant genotypes), and RH0952, RH0555A, RH0644, BPR543-3 (Susceptible genotypes) were selected as parents on the basis of their origin, adaptability, diversity, yield potential, heat tolerance traits. Crosses were attempted during rabi, 2013-14 in a diallel fashion (excluding reciprocals). Further the $\mathrm{F}_{1} \mathrm{~s}$ were grown during rabi, 2014-15. The eight parents along with $28 \mathrm{~F}_{1} \mathrm{~s}$ were evaluated during rabi, 2014-15 in randomized block design with three replications having plot size of two row of three meter length under two environments (normal and late sown) with two dates of sowing 31.10.2014 (normal environment) and 17.11.2014 (late sown) at oilseed research area of Department of Genetics and Plant Breeding, CCS Haryana Agricultural University, Hisar. The data was recorded on eleven characters, form five competitive plants excluding border plants in each $F_{1 s}$ and parents which were randomly selected from each replication. Oil content was estimated by Sokshlet method (AOAC, 1995). All the recommended cultural practices were followed throughout the crop season to raise a good crop. Following statistical model for combining ability was followed.

$X_{i j}=\mu+g_{i}+g_{j}+s_{i j}+1 / r \sum_{K} e_{i j k}$

Where,

$\mu \quad=$ Population mean

$\mathrm{g}_{\mathrm{i}} \quad=$ General combining ability (gca) effects of $i^{\text {st }}$ parent

$\mathrm{g}_{\mathrm{j}} \quad=$ General combining ability effects of $\mathrm{j}^{\text {th }}$ parent 
$\mathrm{s}_{\mathrm{ij}} \quad=$ Specific combing ability (SCA)

effect of ij ${ }^{\text {th }}$ cross/ hybrid

$\mathrm{e}_{\mathrm{ijk}} \quad=$ Environmental component

pertaining to $\mathrm{ijk} \mathrm{k}^{\text {th }}$ observation

$\mathrm{i}$ and $\mathrm{j}=$ Female \& male parents responsible

for producing $\mathrm{ij}^{\text {th }}$ cross/hybrid

$\mathrm{r} \quad=$ Number of replications

Estimation of the combining ability sum of squares, effects and their testing was done by the procedure given by Griffing (1956).

\section{Results and Discussion}

\section{Estimation of Gene action}

Mean squares due to genotypes and $F_{1} \mathrm{~S}$ revealed significant differences for all the traits in both the environments (normal and late sown), indicating presence of adequate genetic variation among the experimental material and both ges and sca were involved in the genetic expression of studied traits. Mean squares due to parent v/s crosses were also significant for all the traits which depicted presence of heterosis for these traits in the series of crosses, except for days to maturity, plant height, siliquae on main shoot, siliqua length, oil content in normal environment and plant height, number of primary branches per plant, siliqua on main shoot, 1000 seed weight in late sown environment. In the present study higher mean values of the hybrids over parents revealed superiority and presence of sufficient amount of heterosis in $\mathrm{F}_{1} \mathrm{~s}$ in both the environments. Superiority of $\mathrm{F}_{1} \mathrm{~s}$ was also reported by Karthikeyan et al., (2009), Shanthi et al., (2011) in rice and Vaghela et al., (2011) and Arifullah (2013) in Mustard. The mean squares due to gca and sca were significant for most of the traits suggesting the operation of both additive and nonadditive components of gene action in the materials study in both environments, except gca for days to maturity and sca for oil content in timely sown condition. These results exhibited the importance of additive type of variance in the inheritance of most of the traits studied, similar findings were reported by Labana et al., (1978) and Tamber et al., (1991) in Indian mustard. The variance due to sca is higher than the gca for the characters viz., days to maturity, plant height, number of secondary branches per plant, oil content and seed yield per plant indicated that role of non-additive gene action inheritance of these traits. The ratio of variance due to general and specific combining ability was low for all the traits studied in both the environments, which was less than unity for all the traits indicating the predominance of non additive gene action for these traits except siliqua length in timely sown condition. In such cases, a breeding strategy which would enable to utilize maximum proportion of fixable genetic variation (additive and additivexadditive epistasis) as well as non additive genetic components (dominance, additivexdominance and dominancexdominance) would be effective. Similar findings were reported by Gupta et al., (2010). Higher magnitude of gca component $\left(\mathrm{g}_{\mathrm{i}}{ }^{2}\right)$ than sca component $\left(\mathrm{s}_{\mathrm{ij}}{ }^{2}\right)$ was observed for siliqua length in timely sown condition, indicating that this trait was mainly under the control of additive genetic variance. The general predictability ratio was near unity for siliqua length and oil content in timely sown condition, indicative that the performance of $F_{1}$ crosses for these traits can be predicted on the basis of general combining ability effects alone.These findings were also corroborated by earlier findings by Patel et al., (1993), Rao and Gulati (2001) with different set of material.

\section{Estimation of general combining ability} (gca)

In the present study, an overall appraisal of gca effects revealed that RH0735 and 
BPR349-9 in normal environment and RH0116, RH8814 and RH0555A in late sown environment emerged as good general combiners for point to seed yield/plant and most of the yield component characters, thus, these genotypes probably possessed the desirable genes for high temperature tolerance during seed filling period. So these parents shall be included in the breeding program for accumulation of favorable alleles in a single genetic background.

High gca effects are related to additive gene effects or additive $\mathrm{x}$ additive interaction effects (Sprague, 1942) which represent the fixable genetic component of variation. Spragme (1966) reported that when general combining ability effects are significant, additive or additivexadditive gene effects are responsible for the inheritance of that particular trait. Hence these parents could be efficiently used for exploiting seed yield. Similar results were revealed by Patel et al., (2012), Yadava et al., (2012), Singh et al., (2013) and Gami and Chauhan (2013) in Brassica juncea. For Brassica, day to maturity and reduced pant height are desirable traits hence; higher the negative values of gca and sca, better are the genotypes for breeding. In our study, maximum negative gca value was exhibited by the genotype, RH0555A in normal environments and RH0644 in late sown environment exhibited negative significant gca effects and these were considered as good general combiners for early maturity.

The parent RH0952 in normal environments, BPR 349-9 in late sown environment had negative gca effects for plant height and thus, considered desirable for dwarfness. Similarly, parents RH8814 in normal and RH0116 in late sown environment for no. of primary branches per plant and RH0735 in normal and RH0116 had significant desirable gca effects in late sown environment for no. of secondary branches per plant. Kumar et al., (1997) and
Teklewold and Becker (2005) also reported similar results in Indian mustard with a different set of material. The genotype RH0644 in both the environments for main shoot length and RH0644 in normal environment for siliquae on main shoot with significant desirable gca effects were found to be the good general combiners. The good general combiners for number of seeds per siliqua and siliqua length were BPR349-9 and RH0116 in normal environment and RH0735 in late sown (stress) environment as they were associated with desirable gca effects. Parents, RH0555A desirably complemented for 1000 seed weight in both the environments and parent RH0116 also complemented for point to oil content in late sown environment showing significant desirable gca effects. Sheikh and Singh (1998) and Acharya and Swain (2004) obtained desirable gca effects for siliqua length in glossy mutant and Pusa Bahar in Brassica juncea. These results clearly indicated that there is a scope for improving combining ability of parents for attributing traits, as good combiners for seed yield traits, therefore, one should breed to improve the combining ability of yield contributing traits which would ultimately improve the gca of seed yield directly.

\section{Estimation of specific combining ability (sca)}

The estimates of SCA are presented in table 4. Crosses, namely, BPR 349-9 x RH0952 in both the environments for early maturity, crosses RH0952 x RH0555A, RH 0735 x BPR 349-9 and RH 8814 x RH 0116 in late sown environment and RH 8814 x RH0555A, RH $0735 \times$ BPR543-3 and RH $0116 \times$ BPR 349-9 in normal environment for dwarfness showed significant negative sca effects. This indicates that the reduction in plant height and days to maturity may be due to negative heterosis in these crosses for these traits, which is desirable. 
Table.1 Analysis of variance for different characters under normal and late sown condition in Indian mustard

\begin{tabular}{|c|c|c|c|c|c|c|c|c|c|c|c|c|}
\hline Source & d.f. & $\begin{array}{c}\text { Days to } \\
\text { maturity }\end{array}$ & $\begin{array}{c}\text { Plant } \\
\text { height } \\
\text { (cm) }\end{array}$ & $\begin{array}{c}\begin{array}{c}\text { No. of 10 } \\
\text { branches/ } \\
\text { plant }\end{array} \\
\end{array}$ & $\begin{array}{c}\text { No. of } 2^{0} \\
\text { branches/ } \\
\text { plant }\end{array}$ & $\begin{array}{c}\text { Main shoot } \\
\text { length } \\
\text { (cm) }\end{array}$ & $\begin{array}{c}\text { Siliquae } \\
\text { on main } \\
\text { shoot }\end{array}$ & $\begin{array}{c}\text { No. of } \\
\text { seeds/ } \\
\text { Siliqua }\end{array}$ & $\begin{array}{c}\text { Siliqua } \\
\text { length } \\
\text { (cm) }\end{array}$ & $\begin{array}{c}\text { Seed } \\
\text { yield/ } \\
\text { plant }(\mathrm{g})\end{array}$ & $\begin{array}{c}1000 \text { seed } \\
\text { weight } \\
\text { (g) }\end{array}$ & $\begin{array}{c}\text { Oil conten } \\
(\%)\end{array}$ \\
\hline & & & & & & \multicolumn{3}{|c|}{ Normal environment } & & & & \\
\hline Replications & 2 & 1.512 & 48.176 & 0.34778 & 0.71361 & 17.428 & 11.815 & 1.180 & 0.076 & 3.538 & 0.188 & 0.154 \\
\hline Genotypes & 35 & $7.564^{* *}$ & $258.640 * *$ & $1.62807 * *$ & $13.531 * *$ & $114.421 * *$ & $31.046^{* *}$ & $3.434 * *$ & $0.494^{* *}$ & $28.864 * *$ & $1.311^{* *}$ & 0.202 \\
\hline Parents & 7 & 2.334 & $255.428^{* *}$ & 0.4619 & 0.74357 & $58.148 * *$ & $52.756^{* * *}$ & $8.860^{* * *}$ & 0.847 ** & $16.508^{* * *}$ & $2.096 * *$ & 0.053 \\
\hline Crosses & 27 & $8.974 * *$ & $268.906^{* *}$ & $1.2845^{* *}$ & $16.346 * *$ & $98.647 * *$ & $26.293 *$ & 2.063 & $0.421 * *$ & $27.100 * *$ & $0.990 * *$ & $0.247^{*}$ \\
\hline $\begin{array}{l}\text { Parents v/s } \\
\text { crosses }\end{array}$ & 1 & 5.979 & 3.8211 & $19.068 * *$ & $27.043 * *$ & $934.214 * *$ & 7.403 & 2.493 & 0.004 & $162.971 * *$ & $4.468^{* * *}$ & 0.054 \\
\hline Error & 70 & 1.557 & 82.899 & 0.39073 & 0.636 & 19.025 & 15.761 & 1.323 & 0.075 & 1.714 & 0.129 & 0.157 \\
\hline & & & & & & \multicolumn{3}{|c|}{ Late sown environment } & & & & \\
\hline Replications & 2 & 0.398 & 58.694 & 0.321 & 0.472 & $75.966 * *$ & $115.629 * *$ & 1.654 & 0.067 & 1.718 & 0.074 & 0.0123 \\
\hline Genotypes & 35 & $6.174 * *$ & $291.51 * *$ & $0.822^{* *}$ & $8.266^{* *}$ & $92.300 * *$ & $60.062^{* *}$ & $3.428^{* *}$ & $0.750^{* *}$ & $29.562 * *$ & $0.568^{* *}$ & $0.534 * *$ \\
\hline Parents & 7 & $4.232 *$ & $330.07 *$ & 0.582 & $5.101^{* *}$ & 14.015 & $171.429 * *$ & $2.685^{* *}$ & $1.513 * *$ & $14.995 * *$ & 0.136 & $0.617^{* *}$ \\
\hline Crosses & 27 & $6.392 * *$ & $286.53 *$ & $0.883^{* *}$ & $8.662 * *$ & $113.391 * *$ & $31.978^{*}$ & $3.080^{* * *}$ & $0.491 * *$ & $31.759 * *$ & $0.697 * *$ & $0.514 * *$ \\
\hline $\begin{array}{l}\text { Parents v/s } \\
\text { crosses }\end{array}$ & 1 & $13.905 * *$ & 156.214 & 0.838 & $19.702 * *$ & $70.850 * *$ & 38.764 & $18.049 * *$ & $2.420^{* *}$ & $72.198 * *$ & 0.089 & 0.489 \\
\hline Error & 70 & 1.579 & 147.923 & 0.340 & 0.371 & 8.556 & 11.548 & 0.824 & 0.124 & 2.546 & 0.104 & 0.125 \\
\hline
\end{tabular}

$*$,** significant at $\mathrm{P}=0.05$ and 0.01 , respectively.

Table.2 Analysis of variance for combining ability for different characters under normal and late sown condition in Indian mustard (Griffing's Method 2, Model I)

\begin{tabular}{|c|c|c|c|c|c|c|c|c|c|c|c|c|c|}
\hline \multirow[t]{2}{*}{ Source } & \multirow[t]{2}{*}{ d.f. } & \multirow{2}{*}{ Environment } & \multicolumn{11}{|c|}{ Mean squares } \\
\hline & & & Days to maturity & $\begin{array}{c}\text { Plant } \\
\text { height } \\
(\mathrm{cm})\end{array}$ & $\begin{array}{c}\text { No. of } 1^{0} \\
\text { branches/ } \\
\text { plant }\end{array}$ & $\begin{array}{c}\text { No. of } 2^{0} \\
\text { branches/ } \\
\text { plant }\end{array}$ & $\begin{array}{l}\text { Main shoot } \\
\text { length } \\
(\mathrm{cm})\end{array}$ & $\begin{array}{c}\text { Siliquae } \\
\text { on main } \\
\text { shoot }\end{array}$ & $\begin{array}{l}\text { No. of } \\
\text { seeds/ } \\
\text { Siliqua }\end{array}$ & $\begin{array}{c}\text { Siliqua } \\
\text { length } \\
(\mathrm{cm})\end{array}$ & $\begin{array}{c}\text { Seed } \\
\text { yield/ } \\
\text { plant } \\
(\mathrm{g})\end{array}$ & $\begin{array}{c}1000 \text { seed } \\
\text { weight } \\
\text { (g) }\end{array}$ & $\begin{array}{c}\text { Oil content } \\
(\%)\end{array}$ \\
\hline \multirow[t]{2}{*}{ Gca } & 7 & Normal & 1.02 & $77.11^{*}$ & $0.63 * *$ & $5.56 * *$ & 18.49 ** & $14.89 *$ & $1.89 * *$ & $0.53 * *$ & $7.15^{* *}$ & $1.03 * *$ & $0.10^{*}$ \\
\hline & & Late & $2.10 * *$ & $109.57^{*}$ & $0.37 * *$ & $2.24 * *$ & $24.44 * *$ & $11.97 * *$ & $1.35 * *$ & $0.70 * *$ & $4.79 * *$ & $0.43 * *$ & $0.16^{*}$ \\
\hline \multirow[t]{2}{*}{ Sca } & 28 & Normal & $2.88^{* * *}$ & 88.49 ** & $0.52 * *$ & $4.25 * *$ & $43.05 * *$ & $9.21 *$ & $0.95 * *$ & $0.07 * *$ & $10.23^{* * *}$ & $0.29 * *$ & 0.06 \\
\hline & & Late & $2.04 * *$ & $94.06^{*}$ & $0.24 * *$ & $2.88 * *$ & $32.34 * *$ & $22.03 * *$ & $0.96 * *$ & $0.13^{* *}$ & $11.11 * *$ & $0.12 * *$ & $0.18^{* * *}$ \\
\hline \multirow[t]{2}{*}{ Error } & 70 & Normal & 0.52 & 27.63 & 0.13 & 0.21 & 6.34 & 5.25 & 0.44 & 0.03 & 0.57 & 0.04 & 0.05 \\
\hline & & Late & 0.52 & 49.30 & 0.11 & 0.12 & 2.85 & 3.84 & 0.27 & 0.904 & 0.84 & 0.03 & 0.04 \\
\hline
\end{tabular}

$*, * *$ significant at $\mathrm{P}=0.05$ and 0.01 , respectively. 
Table.3 Components of combining ability, gca/sca ratio, heritability and general predictability ratio in diallel using Method 2, Model I (Griffing, 1956)

\begin{tabular}{|c|c|c|c|c|c|c|c|c|c|c|c|c|}
\hline Components & Environment & $\begin{array}{l}\text { Days to } \\
\text { maturity }\end{array}$ & $\begin{array}{c}\text { Plant } \\
\text { height } \\
(\mathbf{c m})\end{array}$ & $\begin{array}{c}\text { No. of } \mathbf{1}^{\mathbf{0}} \\
\text { branches/ } \\
\text { plant }\end{array}$ & $\begin{array}{l}\text { No. of } 2^{0} \\
\text { branches/ } \\
\text { plant }\end{array}$ & $\begin{array}{c}\text { Main } \\
\text { shoot } \\
\text { length } \\
\text { (cm) }\end{array}$ & $\begin{array}{c}\text { Siliquae } \\
\text { on main } \\
\text { shoot }\end{array}$ & $\begin{array}{c}\text { No. of } \\
\text { seeds/ } \\
\text { Siliqua }\end{array}$ & $\begin{array}{c}\text { Siliqua } \\
\text { length } \\
(\mathrm{cm})\end{array}$ & $\begin{array}{c}\text { Seed } \\
\text { yield/ } \\
\text { plant } \\
(\mathrm{g})\end{array}$ & $\begin{array}{c}1000 \\
\text { seed } \\
\text { weight } \\
\text { (g) }\end{array}$ & $\begin{array}{c}\text { Oil } \\
\text { content } \\
(\%)\end{array}$ \\
\hline \multirow{2}{*}{$1 / 7 \sum_{1} g_{i}^{2}$} & Normal & 0.055 & 4.947 & 0.050 & 0.535 & 1.215 & $0 . .964$ & 0.145 & 0.050 & 0.657 & 0.099 & 0.005 \\
\hline & Late & 0.157 & 6.027 & 0.025 & 0.211 & 2.15 & 0.812 & 0.108 & 0.066 & 0.394 & 0.040 & 0.012 \\
\hline \multirow{2}{*}{$1 / 28 \sum \sum \mathrm{s}_{\mathrm{ij}}^{2}$} & Normal & 2.365 & 60.85 & 0.390 & 4.035 & 36.710 & 3.957 & 0.515 & 0.048 & 9.667 & 0.244 & 0.005 \\
\hline & Late & 1.521 & 44.762 & 0.136 & 2.76 & 29.49 & 18.18 & 8.14 & 0.094 & 10.26 & 0.092 & 0.139 \\
\hline \multirow[t]{2}{*}{$\mathrm{h}^{2} \mathrm{~ns}$} & Normal & 0.037 & 0.100 & 0.161 & 0.201 & 0.053 & 0.173 & 0.233 & 0.579 & 0.113 & 0.407 & 0.152 \\
\hline & Late & 0.133 & 0.113 & 0.170 & 0.128 & 0.117 & 0.068 & 0.165 & 0.496 & 0.066 & 0.387 & 0.119 \\
\hline \multirow[t]{2}{*}{$\mathrm{h}^{2} \mathrm{bs}$} & Normal & 0.827 & 0.719 & 0.790 & 0.960 & 0.860 & 0.528 & 0.646 & 0.856 & 0.950 & 0.910 & 0.238 \\
\hline & Late & 0.777 & 0.535 & 0.623 & 0.962 & 0.922 & 0.837 & 0.789 & 0.846 & 0.928 & 0.832 & 0.797 \\
\hline \multirow[t]{2}{*}{ gca/sca } & Normal & 0.023 & 0.081 & 0.128 & 0.132 & 0.033 & 0.243 & 0.282 & 1.047 & 0.068 & 0.405 & 0.888 \\
\hline & Late & 0.103 & 0.134 & 0.188 & 0.076 & 0.073 & 0.044 & 0.132 & 0.708 & 0.038 & 0.435 & 0.087 \\
\hline \multirow{2}{*}{$\begin{array}{l}\text { General } \\
\text { predictability } \\
\text { ratio }\end{array}$} & Normal & 0.042 & 0.0139 & 0.204 & 0.209 & 0.062 & 0.327 & 0.352 & 0.677 & 0.119 & 0.447 & 0.639 \\
\hline & Late & 0.172 & 0.212 & 0.0 .274 & 0.133 & 0.128 & 0.082 & 0.209 & 0.586 & 0.072 & 0.465 & 0.149 \\
\hline
\end{tabular}


Table.4 Estimates of gca effects for differents characters under normal and late sown condition in Indian mustard (Griffing, 1956)

\begin{tabular}{|c|c|c|c|c|c|c|c|c|c|c|c|c|}
\hline Components & Environment & $\begin{array}{l}\text { Days to } \\
\text { maturity }\end{array}$ & $\begin{array}{l}\begin{array}{l}\text { Plant } \\
\text { height } \\
(\mathrm{cm})\end{array} \\
\end{array}$ & $\begin{array}{l}\text { No. of } \mathbf{1}^{0} \\
\text { branches/ } \\
\text { plant }\end{array}$ & $\begin{array}{l}\text { No. of } 2^{0} \\
\text { branches/ } \\
\text { plant }\end{array}$ & $\begin{array}{l}\text { Main shoot } \\
\text { length } \\
(\mathrm{cm})\end{array}$ & $\begin{array}{l}\text { Siliquae } \\
\text { on main } \\
\text { shoot }\end{array}$ & $\begin{array}{l}\text { No. of } \\
\text { seeds/ } \\
\text { Siliqua } \\
\end{array}$ & $\begin{array}{l}\begin{array}{l}\text { Siliqua } \\
\text { length } \\
\text { (cm) }\end{array} \\
\end{array}$ & $\begin{array}{l}\text { Seed } \\
\text { yield/plant } \\
(\mathrm{g}) \\
\end{array}$ & $\begin{array}{l}1000 \text { seed } \\
\text { weight } \\
\text { (g) }\end{array}$ & $\begin{array}{l}\text { Oil content } \\
(\%)\end{array}$ \\
\hline \multirow[t]{2}{*}{ RH8814 } & Normal & 0.375 & 1.100 & $-0.489 * *$ & $-0.612 * *$ & -1.339 & -0.834 & -0.131 & $-0.290 * *$ & -0.085 & $-0.185 * *$ & $-0.202 * *$ \\
\hline & Late & 0.342 & 0.750 & -0.182 & $-0.346 * *$ & 0.347 & $1.666^{* *}$ & $-0.393^{*}$ & $-0.286 * *$ & $0.781 * *$ & $-0.138 *$ & $-0.126 * *$ \\
\hline \multirow[t]{2}{*}{ RH0735 } & Normal & 0.108 & -1.730 & -0.019 & $0.402 * *$ & -0.204 & $1.836 * *$ & -0.044 & $0.143 * *$ & $1.358 * *$ & 0.065 & 0.127 \\
\hline & Late & 0.208 & -0.517 & -0.088 & 0.158 & $1.533 * *$ & -0.094 & $0.507 * *$ & $0.144 *$ & -0.018 & 0.086 & 0.011 \\
\hline \multirow[t]{2}{*}{ RH0116 } & Normal & -0.292 & 1.730 & 0.038 & $-0.468 * *$ & 0.574 & 0.786 & $-0.767 * *$ & $0.163 * *$ & $0.935 * *$ & 0.052 & 0.031 \\
\hline & Late & $0.575^{* *}$ & 3.517 & $0.368 * *$ & $0.811 * *$ & $-2.167 * *$ & 0.299 & -0.226 & $0.121 *$ & $1.019 * *$ & 0.066 & $0.237 * *$ \\
\hline \multirow[t]{2}{*}{ BPR349-9 } & Normal & 0.175 & -0.133 & 0.078 & -0.195 & -1.129 & 0.262 & $0.543 * *$ & $-0.307 * *$ & $1.178 * *$ & -0.098 & 0.011 \\
\hline & Late & 0.008 & $-5.883 * *$ & -0.178 & $0.699 * *$ & -0.970 & $-2.19 * *$ & $0.461 * *$ & $0.054 *$ & $-0.911 * *$ & $0.146^{*}$ & -0.069 \\
\hline \multirow[t]{2}{*}{ RH0952 } & Normal & 0.008 & $-5.767 * *$ & 0.187 & $-0.305^{*}$ & $-1.893 *$ & 0.034 & $0.463 *$ & -0.067 & $-0.595 * *$ & -0.005 & 0.074 \\
\hline & Stress & 0.308 & -3.583 & 0.102 & -0.142 & -0.457 & 0.319 & 0.181 & -0.096 & -0.444 & $-0.231 * *$ & $-0.069 *$ \\
\hline \multirow[t]{2}{*}{ RH0555A } & Normal & $-0.592^{* *}$ & 0.567 & $-0.243 *$ & $0.695 * *$ & 0.147 & $-2.26 * *$ & -0.291 & $0.373 * *$ & $0.582 *$ & $0.718 * *$ & -0.076 \\
\hline & Late & -0.125 & 0.083 & -0.112 & -0.119 & $-1.603 * *$ & 0.396 & $-0.369 * *$ & $0.514 * *$ & $0.752 * *$ & $0.386^{* *}$ & $-0.142 *$ \\
\hline \multirow[t]{2}{*}{ RH0644 } & Normal & 0.342 & $3.567 *$ & $0.241^{*}$ & $1.475 * *$ & $1.761 *$ & -0.394 & 0.339 & 0.027 & -0.435 & $-0.212 * *$ & 0.057 \\
\hline & Late & $-0.692 * *$ & 2.517 & 0.155 & -0.136 & $2.260 * *$ & 0.222 & 0.134 & $-0.206 * *$ & 0.043 & $-0.124 *$ & 0.067 \\
\hline \multirow[t]{2}{*}{ BPR543-3 } & Normal & -0.125 & 0.667 & 0.207 & $0.592 * *$ & $1.674^{*}$ & 0.639 & -0.111 & -0.043 & 0.095 & $-0.335 * *$ & -0.023 \\
\hline & Late & $-0.625^{* *}$ & 3.117 & -0.065 & $0.474 * *$ & $1.057 *$ & -0.611 & -0.296 & $-0.246 * *$ & 0.339 & $-0.191 * *$ & 0.091 \\
\hline \multirow[t]{2}{*}{ S.E. (gi) } & Normal & 0.212 & 0.155 & 0.106 & 0.136 & 0.744 & 0.678 & 0.196 & 0.046 & 0.223 & 0.061 & 0.067 \\
\hline & Late & 0.215 & 2.077 & 0.099 & 0.104 & 0.499 & 0.580 & 0.155 & 0.060 & 0.272 & 0.055 & 0.060 \\
\hline \multirow[t]{2}{*}{ S.E. (gi-gj) } & Normal & 0.321 & 2.350 & 0.161 & 0.206 & 1.126 & 1.025 & 0.297 & 0.070 & 0.338 & 0.093 & 0.102 \\
\hline & Late & 0.324 & 3.140 & 0.150 & 0.157 & 0.755 & 0.877 & 0.234 & 0.090 & 0.412 & 0.083 & 0.091 \\
\hline \multirow[t]{2}{*}{ C.D. at $5 \%(g i-g j)$} & Normal & 0.640 & 4.688 & 0.321 & 0.410 & 2.246 & 2.044 & 0.592 & 0.141 & 0.674 & 0.185 & 0.204 \\
\hline & Late & 0.647 & 6.263 & 0.300 & 0.313 & 1.506 & 1.749 & 0.467 & 0.181 & 0.821 & 0.166 & 0.182 \\
\hline \multirow[t]{2}{*}{ C.D. at $1 \%$ (gi-gj) } & Normal & 0.828 & 6.065 & 0.416 & 0.531 & 2.209 & 2.644 & 0.766 & 0.182 & 0.872 & 0.240 & 0.264 \\
\hline & Late & 0.837 & 8.101 & 0.388 & 0.405 & 1.948 & 2.263 & 0.604 & 0.234 & 1.063 & 0.215 & 0.235 \\
\hline
\end{tabular}

\footnotetext{
$*, * *$ significant at $\mathrm{P}=0.05$ and 0.01 , respectively.
} 
Table.5 Estimates of sca effects for different characters under normal and late sown condition in Indian mustard (Griffing, 1956)

\begin{tabular}{|c|c|c|c|c|c|c|c|c|c|c|c|c|}
\hline Components & Environment & Days to maturity & $\begin{array}{c}\text { Plant } \\
\text { height } \\
(\mathbf{c m})\end{array}$ & $\begin{array}{c}\text { No. of } 1^{0} \\
\text { branches/ } \\
\text { plant }\end{array}$ & $\begin{array}{c}{\text { No. of } 2^{0}} \\
\text { branches/ } \\
\text { plant }\end{array}$ & $\begin{array}{l}\text { Main shoot } \\
\text { length } \\
\text { (cm) }\end{array}$ & $\begin{array}{c}\text { Siliquae } \\
\text { on main } \\
\text { shoot }\end{array}$ & $\begin{array}{c}\text { No. of } \\
\text { seeds/ } \\
\text { Siliqua }\end{array}$ & $\begin{array}{c}\text { Siliqua } \\
\text { length } \\
(\mathbf{c m})\end{array}$ & $\begin{array}{c}\text { Seed } \\
\text { yield/ } \\
\text { plant } \\
(\mathrm{g})\end{array}$ & $\begin{array}{l}1000 \text { seed } \\
\text { weight } \\
\text { (g) }\end{array}$ & $\begin{array}{c}\text { Oil conten } \\
(\%)\end{array}$ \\
\hline \multirow[t]{2}{*}{ RH 8814 x RH 0735} & Normal & $-1.881 * *$ & -1.181 & 0.128 & 0.341 & $4.66^{*}$ & 2.139 & 0.097 & -0.050 & 0.289 & 0.126 & -0.154 \\
\hline & Late & $2.163^{* * *}$ & 8.600 & 0.172 & -0.111 & $6.97 * *$ & -2.433 & 0.212 & 0.257 & $2.26^{* * *}$ & 0.014 & 0.016 \\
\hline \multirow[t]{2}{*}{ RH 8814 x RH 0116} & Normal & $-1.148^{*}$ & 0.685 & -0.262 & $1.908^{* *}$ & -1.708 & 0.725 & 0.797 & 0.164 & $4.089^{* *}$ & -0.261 & -0.024 \\
\hline & Late & -0.870 & $-18.4 * *$ & $0.95 * *$ & $1.969^{* *}$ & -2.091 & -2.593 & $1.44 * *$ & $0.58 * *$ & -1.143 & $-0.47^{*}$ & -0.277 \\
\hline \multirow[t]{2}{*}{ RH8814xBPR 349-9 } & Normal & $3.358 * *$ & -2.115 & -0.102 & -0.766 & 0.995 & -2.085 & $1.254^{*}$ & 0.100 & $-2.798 * *$ & 0.189 & 0.296 \\
\hline & Late & -1.304 & 3.967 & -0.271 & 0.113 & $5.51^{*}$ & $5.04 * *$ & 0.925 & -0.153 & $3.987 * *$ & -0.146 & -0.437 \\
\hline \multirow[t]{2}{*}{ RH 8814 x RH0952 } & Normal & $-1.448 *$ & 5.185 & -0.179 & $-1.832 * *$ & 1.325 & -0.121 & 0.001 & 0.227 & -1.118 & 0.229 & 0.100 \\
\hline & Late & 0.396 & 10.667 & 0.182 & $-2.077 * *$ & 2.632 & $-3.73^{*}$ & $-1.26^{* *}$ & -0.236 & 0.420 & 0.364 & 0.129 \\
\hline \multirow[t]{2}{*}{ RH8814 xRH0555A } & Normal & -1.181 & $-12.15 *$ & -0.082 & -0.066 & $9.38 * *$ & $6.44 * *$ & 0.821 & -0.113 & 6.042** & -0.161 & -0.017 \\
\hline & Late & -0.504 & -0.333 & 0.429 & $2.699 * *$ & $6.88 * *$ & 2.043 & 0.455 & 0.121 & $3.524 * *$ & -3.086 & -0.331 \\
\hline \multirow[t]{2}{*}{ RH 8814 x RH0644 } & Normal & $3.885 * *$ & $21.52 * *$ & $-1.03^{* * *}$ & $-1.27 * *$ & $-4.56^{*}$ & -3.191 & -0.409 & -0.233 & $-2.738 * *$ & $0.44 *$ & -0.117 \\
\hline & Late & $2.396^{* * *}$ & 5.900 & -0.371 & $-0.82 *$ & $-9.22 * *$ & -0.317 & -0.248 & $0.51 * *$ & \begin{tabular}{|l|}
$-1.96^{*}$ \\
\end{tabular} & 0.324 & $-.47 *$ \\
\hline \multirow[t]{2}{*}{ RH8814x BPR543-3 } & Normal & 0.685 & $12.75^{* *}$ & -0.332 & -0.686 & 3.292 & 0.072 & 1.241 & $-0.26^{*}$ & $3.959 * *$ & 0.226 & $-0.704 *$ \\
\hline & Late & $-3.004 * *$ & 0.967 & -0.285 & -0.627 & $-7.214 * *$ & 0.883 & -0.085 & -0.119 & -2.130 & 0.056 & $0.40 *$ \\
\hline \multirow[t]{2}{*}{ RH $0735 \times$ RH 0116} & Normal & $-1.548^{*}$ & $9.85^{*}$ & 0.368 & $1.00^{*}$ & 2.615 & 0.152 & -0.523 & 0.130 & 0.039 & 0.189 & -0.087 \\
\hline & Late & 0.596 & 3.167 & $0.75^{*}$ & $2.23 * *$ & $-5.24 * *$ & $-3.75^{*}$ & $1.18^{*}$ & 0.351 & $4.160 * *$ & $-0.46^{*}$ & 0.419 \\
\hline \multirow[t]{2}{*}{ RH0735xBPR 349-9 } & Normal & -0.681 & -1.948 & -0.206 & $0.99 *$ & $8.12 * *$ & 2.009 & -0.766 & 0.034 & \begin{tabular}{|l|}
$-1.98 * *$ \\
\end{tabular} & 0.306 & -0.167 \\
\hline & Late & 0.163 & $18.77 * *$ & -0.165 & -0.257 & -0.741 & -0.883 & 0.759 & 0.117 & \begin{tabular}{|l|}
$-4.543 * *$ \\
\end{tabular} & -0.236 & -0.041 \\
\hline \multirow[t]{2}{*}{ RH 0735 x RH0952 } & Normal & $3.152 * *$ & $\begin{array}{l}-1.315 \\
\end{array}$ & -0.549 & $-2.142 * *$ & $-4.55^{*}$ & 0.872 & -0.753 & -0.140 & -0.335 & $\begin{array}{l}-0.288 \\
\end{array}$ & 0.136 \\
\hline & Late & $1.530^{*}$ & 3.600 & -0.311 & $-0.75^{*}$ & -1.021 & 0.653 & 0.072 & -0.199 & 0.557 & 0.007 & $-0.71 * *$ \\
\hline \multirow[t]{2}{*}{ RH0735x RH0555A } & Normal & 0.752 & -2.315 & $-0.69 *$ & $-3.276 * *$ & -2.625 & -0.435 & 0.234 & 0.087 & $-2.808 * *$ & -0.011 & -0.047 \\
\hline & Late & $-1.704 *$ & 3.933 & -0.231 & $-1.104 * *$ & $-6.41 * *$ & $-6.28 * *$ & $-1.48^{* *}$ & $-0.48^{*}$ & $-7.206^{* *}$ & $0.39 *$ & 0.033 \\
\hline \multirow[t]{2}{*}{ RH 0735 x RH0644 } & Normal & 0.152 & $10.35^{*}$ & -0.269 & 0.188 & $11.56^{* *}$ & $4.70^{*}$ & 0.137 & 0.100 & \begin{tabular}{|l|}
$-1.82 * *$ \\
\end{tabular} & $0.312 *$ & 0.186 \\
\hline & Late & -0.137 & 1.167 & -0.298 & $-1.421 * *$ & $5.49^{* *}$ & -1.870 & -0.048 & $0.48^{*}$ & $3.970 * *$ & 0.267 & $-0.48^{*}$ \\
\hline \multirow[t]{2}{*}{ RH 0735xBPR543-3 } & Normal & -0.048 & $-10.75 *$ & -0.569 & -0.029 & $5.315^{*}$ & 1.232 & 0.587 & 0.004 & \begin{tabular}{|l|}
$1.542^{*}$ \\
\end{tabular} & $-0.32 *$ & 0.100 \\
\hline & Late & -0.537 & -3.433 & 0.055 & -1.031 & 1.632 & -1.670 & -0.118 & 0.22 & -0.593 & -0.233 & -0.201 \\
\hline \multirow[t]{2}{*}{ RH0116xBPR 349-9 } & Normal & $1.719 *$ & $-12.42 *$ & -0.496 & $-2.409 * *$ & $-4.151^{*}$ & -2.138 & 0.124 & 0.417 & -0.648 & $\begin{array}{l}-0.181 \\
\end{array}$ & 0.063 \\
\hline & Late & 0.463 & -3.800 & 0.045 & -0.011 & $-5.47 * *$ & $-3.74 *$ & -0.075 & -0.093 & -1.164 & $0.45^{*}$ & $0.67 * *$ \\
\hline \multirow[t]{2}{*}{ RH 0116 x RH0952 } & Normal & 0.219 & -7.448 & -0.572 & $-1.00 *$ & -0.688 & -3.308 & -0.829 & $0.70^{* *}$ & $1.53^{*}$ & $-0.54 * *$ & -0.100 \\
\hline & Late & -0.170 & -5.433 & -0.235 & 0.266 & 1.579 & $-5.84 *$ & $1.21^{*}$ & -0.043 & $2.15^{*}$ & -0.073 & -0.067 \\
\hline \multirow[t]{2}{*}{ RH0116x RH0555A } & Normal & 0.152 & $\begin{array}{l}-9.781 \\
\end{array}$ & 0.191 & -0.542 & 3.239 & -0.715 & $1.16^{*}$ & -0.166 & $1.16^{*}$ & 0.302 & 0.050 \\
\hline & Late & 0.930 & 0.900 & -0.488 & $-1.691 * *$ & 0.492 & $-3.67 *$ & 0.522 & 0.081 & $-2.54 * *$ & -0.023 & 0.106 \\
\hline
\end{tabular}

$*, * *$ significant at $=0.05$ and 0.01 , respectively. 
Int.J.Curr.Microbiol.App.Sci (2017) 6(5): $942-953$

\begin{tabular}{|c|c|c|c|c|c|c|c|c|c|c|c|c|}
\hline Components & Environment & Days to maturity & $\begin{array}{c}\text { Plant } \\
\text { height } \\
(\mathrm{cm})\end{array}$ & $\begin{array}{c}\text { No. of } 1^{0} \\
\text { branches/ } \\
\text { plant }\end{array}$ & $\begin{array}{c}\text { No. of } 2^{0} \\
\text { branches/ } \\
\text { plant }\end{array}$ & $\begin{array}{l}\text { Main shoot } \\
\text { length } \\
\text { (cm) }\end{array}$ & $\begin{array}{c}\begin{array}{c}\text { Siliquae } \\
\text { on main } \\
\text { shoot }\end{array}\end{array}$ & $\begin{array}{l}\text { No. of } \\
\text { seeds/ } \\
\text { Siliqua }\end{array}$ & $\begin{array}{c}\text { Siliqua } \\
\text { length } \\
(\mathrm{cm})\end{array}$ & $\begin{array}{c}\text { Seed } \\
\text { yield/ } \\
\text { plant } \\
\text { (g) }\end{array}$ & $\begin{array}{l}1000 \text { seed } \\
\text { Weight } \\
\text { (g) }\end{array}$ & $\begin{array}{c}\text { Oil } \\
\text { content } \\
(\%)\end{array}$ \\
\hline \multirow[t]{2}{*}{ RH $0116 \times$ RH0644 } & Normal & 0.552 & 0.219 & -0.092 & $-1.979 * *$ & $-4.47 *$ & 0.489 & 0.16 & 0.080 & $-1.42 *$ & -0.068 & 0.116 \\
\hline & Late & $-1.504 *$ & -6.200 & -0.055 & $1.093^{* *}$ & 1.629 & 0.937 & $-1.12 *$ & -0.233 & $2.83 * *$ & -0.087 & 0.129 \\
\hline \multirow[t]{2}{*}{ RH 0116 x BPR543-3 } & Normal & -0.315 & $10.12^{*}$ & $-1.09 * *$ & $-1.896 * *$ & $9.75 * *$ & 1.719 & 0.31 & $-0.28^{*}$ & -0.291 & 0.289 & -0.237 \\
\hline & Late & -0.570 & 3.533 & -0.035 & -0.417 & 0.599 & 0.203 & 0.08 & 0.207 & $3.50^{* *}$ & 0.354 & $0.57 * *$ \\
\hline \multirow[t]{2}{*}{ BPR 349-9 x RH0952 } & Normal & $-1.581^{*}$ & 5.085 & $-1.08 * *$ & $-2.416 * *$ & $6.32 * *$ & -0.218 & -0.41 & 0.044 & $-2.39 * *$ & $-0.791 * *$ & -0.047 \\
\hline & Late & $-2.604 * *$ & 7.967 & -0.255 & $0.78 *$ & $-5.05^{* *}$ & -2.677 & $1.72 * *$ & $0.82^{* * *}$ & 0.450 & -0.020 & 0.073 \\
\hline \multirow[t]{2}{*}{ BPR 349-9 x RH 0555A } & Normal & 0.019 & -3.915 & $-1.25 * *$ & $-1.33^{* *}$ & $-9.591 * *$ & $-6.76 * *$ & $-1.95 * *$ & 0.104 & $1.47^{*}$ & $0.32 *$ & -0.097 \\
\hline & Late & $-1.504 *$ & 0.700 & $-0.77 *$ & $-1.247 * *$ & $-3.44 *$ & -0.640 & $-0.96^{*}$ & 0.114 & $-2.78^{* * *}$ & $0.63 * *$ & 0179 \\
\hline \multirow[t]{2}{*}{ BPR 349-9 x RH0644 } & Normal & 0.248 & -3.915 & 0.534 & $1.714^{* *}$ & 2.895 & 0.679 & -0.22 & -0.116 & $5.192^{* *}$ & $-0.32^{*}$ & -0.164 \\
\hline & Late & 1.063 & $15.53^{*}$ & 0.592 & $-1.297 * *$ & $5.99 * *$ & 3.067 & -0.47 & $-0.43 *$ & $4.264 * *$ & -0.093 & 0.103 \\
\hline \multirow[t]{2}{*}{ BPR 349-9 x BPR543-3 } & Normal & 0.885 & 8.319 & $1.00^{* *}$ & $1.03^{*}$ & -1.018 & 3.342 & $1.53 *$ & 0.187 & $3.555^{* *}$ & -0.149 & 0.150 \\
\hline & Late & 0.996 & 7.933 & $0.51 *$ & $2.659^{* * *}$ & $5.40^{* * *}$ & $8.60^{* *}$ & -0.71 & -0.126 & $3.07 * *$ & -0.126 & 0.221 \\
\hline \multirow[t]{2}{*}{ RH0952 x RH0555A } & Normal & $1.519^{*}$ & -2.281 & -0.092 & 0.584 & -2.261 & 1.872 & 0.26 & 0.130 & $3.052^{* * *}$ & 0.308 & -0.327 \\
\hline & Late & 0.863 & $-20.67 * *$ & -0.188 & 0.429 & 1.249 & -0.037 & 0.25 & 0.164 & $2.65^{* *}$ & $-0.41 *$ & 0.346 \\
\hline \multirow[t]{2}{*}{ RH0952 x RH0644 } & Normal & -0.415 & -8.615 & 0.091 & 1.948 ** & $-4.44 *$ & $-5.03^{*}$ & 0.33 & -0.123 & $1.44 *$ & $-1.0^{* * *}$ & 0.206 \\
\hline & Late & -0.904 & -4.100 & -0.021 & -0.521 & $11.15^{* * *}$ & 3.237 & -0.25 & 0.051 & $-2.97 * *$ & $-0.75^{* *}$ & 0.069 \\
\hline \multirow[t]{2}{*}{ RH0952 x BPR543-3 } & Normal & $1.385^{*}$ & 2.619 & 0.558 & $3.898^{* * *}$ & $5.945^{*}$ & 1.505 & -0.75 & $-0.52 * *$ & -0.965 & $-0.65 * *$ & $0.59^{* * *}$ \\
\hline & Late & -1.304 & 3.300 & 0.099 & $-0.83^{*}$ & $-6.211 * *$ & $-3.93 *$ & 0.142 & 0.157 & -0.166 & $-0.45^{*}$ & -0.187 \\
\hline \multirow[t]{2}{*}{ RH0555A x RH0644 } & Normal & -0.148 & -4.615 & 0.154 & $2.748^{* * *}$ & $6.12 * *$ & 1.102 & -0.949 & -0.030 & 0.232 & -0.010 & -0.110 \\
\hline & Late & -0.137 & 3.233 & $1.06 * *$ & $3.756^{* *}$ & $-6.23 * *$ & $4.41 *$ & $2.462 * *$ & -0.126 & $4.734^{* * *}$ & -0.133 & -0.157 \\
\hline \multirow{2}{*}{ RH0555A x BPR543-3 } & Normal & -0.681 & 7.619 & -0.446 & $-3.436^{* *}$ & 3.27 & -0.57 & 0.134 & 0.074 & -1.105 & $-0.38^{*}$ & 0.103 \\
\hline & Late & 0.130 & $13.97 *$ & -0.255 & $1.013^{* * *}$ & 9.60 ** & $3.98^{*}$ & 0.159 & -0.253 & 0.007 & -0.166 & 0.053 \\
\hline \multirow{2}{*}{ RH0644 x BPR543-3 } & Normal & $-3.615 * *$ & $-12.38^{*}$ & 0.071 & $1.294 * *$ & -0.71 & -0.56 & -0.163 & $-0.31 *$ & $5.215^{* *}$ & $-0.38^{*}$ & -0.030 \\
\hline & Late & -0.304 & -3.467 & $0.71 *$ & $2.596 * *$ & $3.64 *$ & 2.04 & $1.35 * *$ & $0.50^{* * *}$ & $-3.886 * *$ & $-0.32 *$ & $-0.72^{* * *}$ \\
\hline \multirow[t]{2}{*}{ S.E. $\left(S_{\mathrm{ii}}\right)$} & Normal & 0.566 & 4.146 & 0.284 & 0.363 & 1.986 & 1.81 & 0.523 & 0.124 & 0.596 & 0.164 & 0.180 \\
\hline & Late & 0.572 & 5.438 & 0.265 & 0.277 & 1.332 & 1.547 & 0.413 & 0.160 & 0.726 & 0.147 & 0.161 \\
\hline \multirow[t]{2}{*}{ S.E. $\left(S_{i j}\right)$} & Normal & 0.651 & 4.766 & 0.327 & 0.417 & 2.283 & 2.078 & 0.602 & 0.143 & 0.685 & 0.188 & 0.207 \\
\hline & Late & 0.657 & 6.367 & 0.305 & 0.318 & 1.531 & 1.779 & 0.476 & 0.184 & 0.835 & 0.169 & 0.185 \\
\hline \multirow[t]{2}{*}{ S.E. $\left(S_{i i}-S_{i j}\right)$} & Normal & 0.786 & 5.758 & 0.395 & 0.504 & 2.758 & 2.510 & 0.727 & 0.173 & 0.828 & 0.227 & 0.250 \\
\hline & Late & 0.794 & 7.692 & 0.369 & 0.385 & 1.849 & 2.149 & 0.574 & 0.222 & 1.009 & 0.204 & 0.223 \\
\hline \multirow[t]{2}{*}{ S.E. $\left(\mathrm{S}_{\mathrm{ij}} \mathrm{S}_{\mathrm{ik}}\right)$} & Normal & 0.963 & 7.052 & 0.484 & 0.618 & 3.378 & 3.075 & 0.891 & 0.212 & 1.014 & 0.279 & 0.307 \\
\hline & Late & 0.973 & 9.420 & 0.451 & 0.471 & 2.265 & 2.632 & 0.703 & 0.272 & 1.236 & 0.250 & 0.273 \\
\hline \multirow[t]{2}{*}{ S.E. $\left(S_{i j}-S_{k 1}\right)$} & Normal & 0.908 & 6.649 & 0.457 & 0.582 & 3.185 & 2.899 & 0.840 & 0.200 & 0.956 & 0.263 & 0.289 \\
\hline & Late & 0.917 & 8.882 & 0.426 & 0.444 & 2.136 & 2.481 & 0.663 & 0.257 & 1.165 & 0.236 & 0.258 \\
\hline \multirow[t]{2}{*}{ C.D. at $5 \%\left(\mathrm{~S}_{\mathrm{ij}}-\mathrm{S}_{\mathrm{ik}}\right)$} & Normal & 1.921 & 14.065 & 0.965 & 1.232 & 6.738 & 6.133 & 1.777 & 0.423 & 2.022 & 0.556 & 0.612 \\
\hline & Late & 1.941 & 18.789 & 0.901 & 0.941 & 4.518 & 5.249 & 1.402 & 0.543 & 2.465 & 0.499 & 0.546 \\
\hline \multirow[t]{2}{*}{ C.D. at $1 \%$} & Normal & 2.485 & 18.195 & 1.249 & 1.594 & 8.710 & 7.934 & 2.298 & 0.547 & 2.616 & 0.720 & 0.792 \\
\hline & Late & 2.511 & 24.305 & 1.166 & 1.217 & 5.845 & 6.791 & 1.814 & 0.701 & 3.189 & 0.646 & 0.707 \\
\hline
\end{tabular}

****ignificant at $=0.05$ and 0.01 , respectively. 
The results are in accordance with, Nasrin et al., (2011), Gupta et al., (2011), Vaghela et al., (2011) identified superior cross combinations on the basis of gca and sca effects for days to maturity and plant height in mustard.

Cross combination, i.e., BPR 349-9 $\mathrm{x}$ BPR543-3, RH 8814 x RH 0116, RH 0735 x RH 0116, BPR 349-9 x BPR543-3, RH0555A x RH0644 and RH0644 x BPR543-3 for no. of secondary branches per plant, RH 8814 x RH 0735, RH 8814 x RH0555A and RH 0735 $\mathrm{X}$ RH0644 for main shoot length showed significant positive sca effects in both the environments. Crosses, RH 0735 x RH0644 in normal environment and BPR 349-9 $\mathrm{x}$ BPR543-3, RH 8814 x BPR 349-9 in late sown environment for siliquae on main shoot, BPR349-9xBPR543-3, RH 0116 x RH0952 in normal environment and RH $8814 \times \mathrm{RH}$ 0116, BPR 349-9 x RH0952 in late sown environment for number of seeds per siliqua and siliqua length were expressed significant highest positive sca effects. Our findings are in agreement with the earlier results in mustard reported by Singh and Murty (1980), Sheikh and Singh (1998) and Chowdhury et al., (2004a).The magnitude of sca effects for seed yield/plant revealed in crosses viz., RH 8814 x RH0555A, RH 0116 x RH0952, BPR 349-9 x RH0644, BPR 349-9 x BPR543-3 and RH0952 $x$ RH0555A in both the environments, seven crosses in normal and late sown environment showed significant positive sca effects. Teklewold et al., (2005), Nassimi et al., (2006) and Wang et al., (1997) observed significant positive sca effect for seed yield. Significant positive sca effects in both the environments were expressed by only one cross combinations, namely, BPR 349-9 x RH0555A for 1000 seed weight, RH0952 x BPR543-3 in normal and RH 8814 $x$ BPR543-3, RH 0116 x BPR 349-9 and RH 0116 x BPR543-3 in late sown environments oil content. The outcomes clearly indicate that the parents involved in these crosses were good specific combiners; however, the relative contribution of the parents to specific combining ability effect for seed yield was through various yield attributing traits in different hybrids (Tables 1-5). Singh et al., (2000) in YSC-68 X SS-2 in Brassica campestris, Chowdhury et al., (2004a) in Dhali x Sampad in Brassica rapa, Acharya and Swain (2004) in Pusa Bold x Pusa Bahar in Brassica juncea observed significant positive sca effects for 1000 seed weight. Sheikh and Singh (1998) obtained significant positive sca effects for oil content in Glossy mutant x BJ-1257 and poorbijaya x BJ-38 respectively in Brassica juncea. These crosses and parent could be extensively used in breeding programme to develop superior segregants could be derived in further breeding programmes.

\section{References}

Acharya, N.N. and Swain, D. 2004. Combining ability analysis of seed yield and its components in Indian Mustard (B. juncea L.). Ind. J. Agric. Res., 38(1): 40-44.

Amiri-Oghana, H., Fotokianb, M.H., Javidfar F. and Alizadeha, B. 2009. Genetic analysis of seed yield, days to flowering and maturity in oilseed rape (Brassica napus L.) using diallel crosses. Int. J. Pl. Production, 2:19-26.

AOAC. 1995. Official methods of analysis, $\left(11^{\text {th }}\right.$ Eds) Association of official agricultural chemists, Washington D.C.

Arifullah, M., Munir, M., Mahmood, A., Ajmal, K.S. and Hassan-ul-F. 2013. Genetic analysis of some yield attributes in Indian Mustard (Brassica juncea L.) Afri. J. Pl. Sc., 7(6): 219-226.

Chowdhury, M.A.Z., Mian, M.A.K., Akbar, M.A. and Alam, M.Z. 2004a. Combining ability for seed yield and yield contributing characters in turnip rape (Brassica rapa L.). Bangladesh $J$. 


\author{
Pl. Breed. Genet., 17(1): 17-24. \\ FAO. $2013 . \quad$ FAOSTAT-2011. \\ http://www.fao.org. (July 17, 2013).
}

Gami, R.A. and Chauhan, R.M. 2013. Heterosis and Combining Ability Anlaysis for Seed Yield and its attributes in Indian Mustard [Brassica juncea (L.) Czern and Coss.] Indian J. Agri. Res., 47(6): 535-539.

Griffing, B. 1956. Concept of general and specific combining ability in relation to diallel systems. Aust. J. Biol. Sci., 9: 463-493.

Gupta, P., Chaudhary, H.B., Lal Kumar, S. 2010. Heterosis and combining ability analysis for yield and its components in Indian mustard (Brassica juncea L. Czern and Coss) Front. Agric. China, 4(3): 299-307

Karthikeyan P., Anbuselvam Y., Palaniraja K., Elangaimannan R. 2009. Combining ability of rice genotypes under coastal saline soils. Elect. J. Plant Breed., 1: $18-23$.

Kumar, P., T.P. Yadav, L. Raj, S.K. Gupta, N.K. Thakral, P. Kumar and L. Raj, 1997. Combining ability and heterosis for oil content in toria (B. campestris). Cruciferae Newslett., 19: 87-88.

Labana, K.S., Jindal, S.K. and Menon, D.K. 1978. Heterosis and combining ability in yellow sarson. Crop Improvement, 2: 46-51.

Leon, J. 1991. Heterosis and mixing effects in winter oilseed rape. Crop Sci., 31: 281284.

Nasrin, S., F. Nur, M.K. Nasreen, M.S.R. Bhuiyan, S. Sarkar and M.M. Islam. 2011. Heterosis and combining ability analysis in Indian mustard (B. juncea L.). Bang. Res. Pub. J., 6(1): 65-71.

Nassimi, A.W., Ali, R.S., Hassan, G. and Ali, N. 2006. Combining ability analysis for maturity and other traits in rapeseed (Brassica napus). J. Agron., 5(3): 523526.
Patel, A.M., Prajapati, D.B. and Patel, D.G. 2012. Heterosis and combining ability studies in Indian Mustard (Brassica juncea L.) Ind. J. Sci. Res. and Tech., 1(1): 38-40.

Patel, K.M., Prajapati, K.P., Fatteh, U.G. and Patel, I.D. 1993. Combining ability and heterosis in Indian mustard. J. Oilseed Res, 9(1): 169-174.

Ramsay, L.D., Bradshaw, J.E., and Kearsey, M.J. 1994. The inheritance of quantitative traits in Swedes (Brassica napus spp. rapifera) diallel analysis of dry matter yield. J. Genet. Breed, 48: 253-257.

Rao, N.V.P.R.G. and Gulati, S.C. 2001. Combining ability of gene action in $\mathrm{F} 1$ and F2 diallels of Indian mustard [Brassica juncea (L.) Czern and Coss.]. J. Crop. Res. Hissar., 21(1): 72-76.

Shanthi, P., Jebaraj, S., Geetha, S. 2011. Study on gene action for sodic tolerance traits in rice (Oryza sativa L.). Elect. J. Plant Breeding, 2(1): 24-30.

Singh, A., Avtar, R., Singh, D., Sangwan, O., Thakral, N. K., Malik, V. S., Goyat, B. and Dalal, U. 2013. Combining Ability Analysis for Seed Yield and Component Traits in Indian Mustard [Brassica juncea(L.) Czern and Coss.]. Res. Plant Biol., 3(2): 26-31.

Singh, J.N. and Murty, B.R. 1980. Combining ability and maternal effects in Brassica campestris (L.) var. Yellow sarson. Theor. Appl. Genet., 56: 265-272.

Singh, J.N., Maheshc, Yadav and Sheikh, I.A. 1998. Genetical studies for yield and oil content in Brassica juncea (L.). Indian J. Genet., 56(3): 299-304.

Singh, M., Singh, L. and Srivastava, S.B.L. 2010. Combining ability analysis in Indian mustard (B. juncea). J. Oilseed Brassica, 1: 23-27.

Singh, M.., Singh, S.P. and Dhirendra, S. 2000. Genetic analysis for yield and its genotypes in yellow sarson (Brassica 
campestris). Indian J. Agric. Sci., 70(9): 624-626.

Spragme, G.F. 1966. Quantitative Genetics in Plant Improvement. In: "Plant Breeding", (Ed.): Fey, K. J.. Iowa State University Press. Iowa, PP. 315-354.

Sprague, G.F. and Tatum, L.A. 1942. General vs specific combining ability in single crosses in corn. J. American Soc. Agron., 30: 923-932.

Tamber, K.S., Singh, M. and Gupta, M.L. 1991. Combining ability for earliness in Indian mustard (Brassica juncea L. Czerny). Indian J. Genetics, 49: 223-6.

Teklewold, A., and Becker, H.C. 2005. Heterosis and combining ability in a diallel cross of Ethiopian Mustard inbred lines. Crop Sci., 45: 2629-2635.

Thakur, H.L., and Sagwal, J.C. 1997. Heterosis and combining ability in oilseed rape (B. napus). Indian $J$. Genet., 57: 163-167.

Vaghela, P.O., Thakkar, D.A., Bhadauria, H.S., Sutariya, D.A., Parmar, S.K. and
Prajapati, D.V. 2011. Heterosis and combining ability for yield and its component traits in Indian mustard [Brassica juncea (L.) Czern and Coss] J. Oilseeds Res., 2(1): 39-43.

Wang, Y., P. Luo, Wang and Luo, P. 1997. Intergenic hybridization between Brassica species and Crambe abyssinica. Rosliny Oleiste, 18: 171178.

Wos, H., Bartkowiak-Broda, I., Budzianowski, G. and Krzymanski, J. 1999. Breeding of winter and spring oilseed rape hybrids at Malyszyn. Paper 544. In Proc. 10th Int. Rapeseed Confr. [CDROM]. 26-29 Sep. 1999, Canberra, Australia.

Yadava, D.K., Singh N., Vasudev S., Singh R., Singh,S., Giri S.C., Dwivedi V.K. and Prabhu K.V. 2012. Combining ability and heterobeltiosis for yield and yield-contributing traits in Indian mustard (Brassica juncea). Indian $J$. Agri. Sci., 82(7): 563-567.

\section{How to cite this article:}

Baldeep Singh, N.K. Thakral, Ram Avtar and Geeta Boken. 2017. Combining Ability Analysis: Morphological Traits for High Temperature Stress Tolerance in Indian Mustard [Brassica juncea (L.) Czern \& Coss.]. Int.J.Curr.Microbiol.App.Sci. 6(5): 942-953. doi: https://doi.org/10.20546/ijcmas.2017.605.104 